\title{
Effect of ultrasonic seed treatment on rice seedlings under waterlogging stress
}

\author{
Suihua Huang ${ }^{1,2}$, Yuanyuan Jia ${ }^{1}$, Ping Liu ${ }^{1}$, Hao Dong ${ }^{1}$, and Xiangru Tang ${ }^{1,2^{*}}$ \\ ${ }^{1}$ South China Agricultural University, College of Agriculture, State Key Laboratory for Conservation and Utilization of Subtropical \\ Agro-bioresources, Guangzhou, 510642, China. \\ ${ }^{2}$ Ministry of Agriculture and Rural Affairs, Scientific Observing and Experimental Station of Crop Cultivation in South China, \\ Guangzhou, 510642, PR China. "Corresponding author (tangxr@scau.edu.cn).
}

Received: 11 April 2020; Accepted: 21 July 2020; doi:10.4067/S0718-58392020000400561

\begin{abstract}
One of the main problems of rice (Oryza sativa L.) cultivation is erratic germination and crop establishment; therefore, a pre-sowing ultrasonic seed treatment was studied to improve this situation. A pot experiment was conducted to investigate the effect of ultrasonic seed treatment on rice seedlings under waterlogging conditions. Two cultivars of rice seeds, 'Yuejingsimiao' (YJSM) and 'Yuxiangyouzhan' (YXYZ), were treated with ultrasonic waves before being transferred to the pot. The ultrasonic treatment included seed treatments in the dry state (S1), wet state (S2), and a control (CK). The waterlogging treatment included three flooding levels, control (W0), 2.0-3.0 cm flooding (W1), and 3.0-4.0 cm flooding (W2). The seedling emergence rate for W1S2 was 35.07\% to $64.39 \%$ higher than W1CK, while fresh weight for W2S2 increased by $54.21 \%$ to $89.98 \%$ compared with W2CK. Furthermore, W1S1 (11.74\% to $15.02 \%)$ and W1S2 (7.87\% to $43.29 \%$ ) also increased catalase activities compared with W1CK. Malondialdehyde (MDA) decreased by $19.87 \%$ to $31.84 \%$ and $9.70 \%$ to $38.74 \%$ for YJSM and YXYZ, respectively. The results showed that under waterlogging conditions, wet seed under ultrasonic treatment significantly increased the seedling emergence rate. Both the wet and dry seed treatments improved seedling quality, antioxidant activity, but decreased the MDA content.
\end{abstract}

Key words: Antioxidants, germination, flooding condition, Oryza sativa, ultrasound.

\section{INTRODUCTION}

Water in a suitable irrigation system is required for plant growth because excessive flooding or submergence damages plants. An enzymatic defense mechanism, including peroxidase (POD) and catalase (CAT), is triggered to inhibit plants from reactive oxygen species (ROS) injury, apparently affecting the plant metabolism (Fernández et al., 2013). When plants are threatened by abiotic stresses, such as waterlogging, the defense mechanism has to scavenge free radicals, depress lipid peroxidation, and reduce malondialdehyde (MDA) production by counterattacking the cell membrane system (Luo et al., 2018).

Poor field drainage and deep underground water lead to a low seedling emergence rate and even stunted rice. Research has previously shown that waterlogging has an impact on seed germination, seedling growth, and crop yield (Ghobadi et al., 2017). Flooding demonstrates slow gaseous diffusion and rapid oxygen expenditure by the rhizosphere microorganism; excess water limits the oxygen supply to the submerged tissues, resulting in plant damage (Nishiuchi et al., 2012). While roots face the danger of waterlogging, tolerant crop genotypes have a greater ability to grow than susceptible genotypes (Sairam et al., 2011). During submergence or various stress conditions, the accumulation of oxygen free radicals caused by stomatal closure functions in photosynthetic cells and limits $\mathrm{CO}_{2}$, thus inhibiting root growth (Srivastava et al., 2009).

Seeds are a crucial material for crop propagation in the future; the loss of emergence ability and vigor causes crops to eventually reduce stand establishment in the field when seeds are in long-term or unsuitable storage (Groot et al., 2012; 
Rissel et al., 2014; Wojtyla et al., 2016). Along with increasing ROS during storage, which is one of the main factors of seed declination, crops with poor germination and stand establishment suffer yield loss (Rissel et al., 2014). Seeds with proper moisture (technically dried) are stored after harvest, but their vigor decreases over time and inevitably age (Groot et al., 2015). Various seed treatment techniques have been chemically and mechanically used to promote seed longevity and seedling emergence and improve seed ability under stress or long-term storage (Du et al., 2019).

Ultrasound is widely used in physical extraction and helps improve plant growth. Therefore, it affects their biology in a complex manner without damaging plant molecules and cells, but promotes their biological synthesis (Leon et al., 2018). An ultrasonic seed pretreatment provides basic conditions for plant growth and has a positive impact on the electrical conductivity and physiological substances in seeds (Wang et al., 2012). In response to stress suffered by rice, yield, quality, and antioxidants were positively enhanced after seeds were subjected to ultrasound treatment (Rao et al., 2018). Germination rate and plant survival have been used in previous studies as characteristics of waterlogging tolerance in barley and its visible response to waterlogging (Miano et al., 2015). Although widely used in engineering, ultrasound is relatively less applied to agriculture; the ultrasonic treatment has been used to investigate crop growth such as in beans, chickpeas, peppers, and rice (Goussous et al., 2010; Rao et al., 2018). Therefore, it is hypothesized that the ultrasound technique improves the germination of seeds that lack vitality and plant regulation could be obtained; the subsequent impact of ultrasound on rice seedlings under waterlogging stress was studied.

\section{MATERIALS AND METHODS}

A pot experiment was conducted at the Experimental Research Farm ( $23^{\circ} 09^{\prime} \mathrm{N}, 113^{\circ} 22^{\prime}$ E), College of Agriculture, South China Agricultural University, Guangzhou, China. Two rice (Oryza sativa L.) cultivars, 'Yuejingsimiao' (YJSM) and 'Yuxiangyouzhan' (YXYZ), were provided by the College of Agriculture, South China Agricultural University, and sown on 10 March 2018 under a double rice cropping system in South China. Ultrasound at $20 \mathrm{kHz}, 220 \mathrm{~W}$ was used to directly treat the dried rice seeds in the ultrasonic seed processor (Guangzhou Xindongli Ultrasound Electronic Equipment, Guangzhou, China). Seeds were divided into three groups before sowing: seeds treated by ultrasonic waves in the dry state (S1), seeds treated by ultrasonic waves in the wet state (S2), and no treatment applied as a control (CK). Flooding treatments included irrigation after sowing with three levels of flooding: no flooding (W0), $2.0-3.0 \mathrm{~cm}$ flooding (W1), and 3.0-4.0 cm flooding (W2).

After the treatment, rice seeds were carefully sown in plastic pots $(31 \mathrm{~cm}$ diameter and $29 \mathrm{~cm}$ height $)$ and grown in a phytotron at $27^{\circ} \mathrm{C} / 21{ }^{\circ} \mathrm{C}$ day/night under 1200 lx yellow light intensity and $75 \%$ humidity. The experimental soil contained $21.03 \mathrm{~g} \mathrm{~kg}^{-1}$ organic matter, $0.75 \mathrm{~g} \mathrm{~kg}^{-1}$ total N, $0.73 \mathrm{~g} \mathrm{~kg}^{-1}$ total $\mathrm{P}, 18.23 \mathrm{~g} \mathrm{~kg}^{-1}$ total $\mathrm{K}, 63.72 \mathrm{mg} \mathrm{kg}^{-1}$ alkali hydrolyzed N, $28.94 \mathrm{mg} \mathrm{kg}^{-1}$ effective $\mathrm{P}$, and $47.61 \mathrm{mg} \mathrm{kg}^{-1}$ available $\mathrm{K}$. Thirty-day-old seedlings were collected to determine seedling growth and physiological parameters, washed, and immediately stored at $-80^{\circ} \mathrm{C}$ until the biochemical analyses.

\section{Measurement of rice growth and SPAD values}

The seedling emergence rate was determined on 26 March and a $10 \times 10 \mathrm{~cm}$ area was chosen to count emerged seedlings as expressed by seedling emergence rate $(\%)=$ (number of emerged seedlings/total number of seeds) $\times 100$. Seedlings were sampled on 10 April in three biological replicates to evaluate fresh weight $(\mathrm{g})$, dry weight $(\mathrm{g})$, plant height $(\mathrm{cm})$, basal diameter $(\mathrm{cm})$, and number of roots. To evaluate fresh weight, plants were weighed immediately after sampling and oven-dried at $80{ }^{\circ} \mathrm{C}$ for $48 \mathrm{~h}$ to obtain dry weight. Leaf chlorophyll fluorescence was measured with a chlorophyll fluorometer (SPAD-502 Plus v. 1.20; Konica Minolta, Chiyoda, Tokyo, Japan).

\section{Antioxidant activity}

Peroxidase (POD, EC1.11.1.7) activity was determined using the method indicated by Ashraf et al. (2017). The reaction mixture contained enzyme extract $(50 \mu \mathrm{L}), 1 \mathrm{~mL} 0.3 \% \mathrm{H}_{2} \mathrm{O}_{2}, 0.95 \mathrm{~mL} 0.2 \%$ guaiacol, and $1 \mathrm{~mL} 50 \mathrm{mmol} \mathrm{L}^{-1} \mathrm{pH} 7.0$ phosphate buffer solution (PBS). The change in absorbance was read at $420 \mathrm{~nm}$ at 30-s intervals up to 2 min. Catalase (CAT, EC 1.11.1.6) activity was determined with the CAS assay kit (Nanjing Jiancheng Bioengineering Institute, Nanjing, China). One unit of CAT activity was estimated as the amount of enzyme that decomposes $1 \mu \mathrm{mol} \mathrm{H}_{2} \mathrm{O}_{2}$ at $405 \mathrm{~nm} \mathrm{~s}^{-1}$ in 1 $\mathrm{mg}$ fresh tissue proteins. 


\section{Malondialdehyde and soluble protein contents}

Malondialdehyde (MDA) content was measured as described by Rao et al. (2018). Fresh leaves $(0.5 \mathrm{~g})$ from the control and treated plants were homogenized in $2 \mathrm{~mL} \mathrm{20 \%} \mathrm{(w/v)} \mathrm{trichloroacetic} \mathrm{acid} \mathrm{(TCA).} \mathrm{Afterward,} 1.5 \mathrm{~mL} 20 \%$ TCA containing $0.5 \%(\mathrm{w} / \mathrm{v}) \mathrm{TBA}$ and $100 \mu \mathrm{L} 4 \%$ butylated hydroxytoluene (BHT) in ethanol was added to the aliquot $(0.5 \mathrm{~mL})$ of the supernatant. This was heated at $100{ }^{\circ} \mathrm{C}$ for $30 \mathrm{~min}$ and quickly cooled in ice. The homogenate was centrifuged at $3000 \mathrm{~g}$ for $15 \mathrm{~min}$. After cooling the reaction mixture was measured at 450,532 and $600 \mathrm{~nm}$ and the unit of MDA content was $\mu \mathrm{mol} \mathrm{g} \mathrm{g}^{-1} \mathrm{FW}$.

Soluble protein content was evaluated following the method described by Rao et al. (2018). Fresh leaves $(0.5 \mathrm{~g})$ were homogenized in $9 \mathrm{~mL}$ phosphate buffer at $\mathrm{pH} 7.8$, centrifuged at $8000 \mathrm{rpm}$ at $4{ }^{\circ} \mathrm{C}$ for $15 \mathrm{~min}$. Afterward, $100 \mu \mathrm{L}$ supernatant was mixed with ultrapure water $(900 \mu \mathrm{L})$ and Coomassie Brilliant Blue $\mathrm{G} 250$ reagent, and kept at $25^{\circ} \mathrm{C}$ after mixing for $20 \mathrm{~min}$. Absorbance was tested at $595 \mathrm{~nm}$.

\section{Statistical analysis}

The experiment was conducted using a completely randomized block design with three replicates of each treatment. Data collected were statistically analyzed by ANOVA with the Statistix 8 statistical software (Analytical Software, Tallahassee, Florida, USA). The differences among treatments were separated according to the least significant difference (LSD) test at $\mathrm{P}<0.05$. Multivariate analysis was preceded by MetaboAnalyst (Xia Lab, McGill University, Ste. Anne de Bellevue, Quebec, Canada; http://www.metaboanalyst.ca).

\section{RESULTS}

\section{Effect of ultrasonic treatment on seedling emergence rate}

The ultrasonic treatment significantly affected the seedling emergence rate under waterlogging stress in both cultivars $(\mathrm{P}<0.05$; Figure 1). For YJSM, there were significant differences between S2 and CK under W1 and W2, whereas nonsignificant differences were detected under W0. The seedling emergence rate for W1S2 was $63.49 \%$ higher than W1CK, while W2S2 was 39.39\% higher than W2CK. A similar trend was observed in YXYZ in which S2 could significantly enhance the seedling emergence rate under W1 and W2; both WOS1 (9.33\%) and WOS2 (14.22\%) were higher than W0CK. The W1S2 treatment significantly enhanced the seedling emergence rate by $28.21 \%$ compared with W1CK, while W2S1 and W2S2 were $20.15 \%$ and $35.07 \%$ higher than in W2CK.

Figure 1. Effect of ultrasonic and waterlogging treatments on seedling emergence rate.

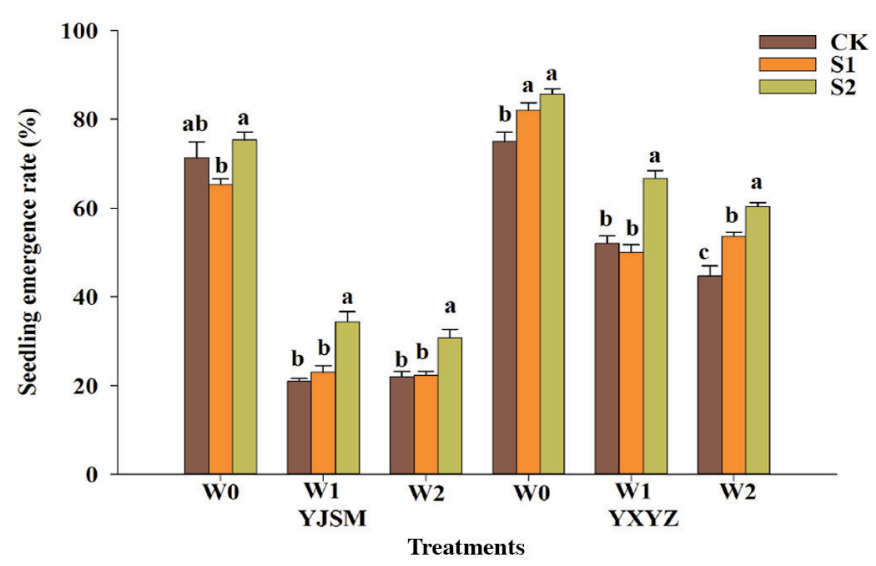

Error bars above means were the standard error of three replicates. Different letters over bars for a treatment were significant according to the LSD test $(\mathrm{P}<0.05)$.

YJSM: Yuejingsimiao; YXYZ: Yuxiangyouzhan; W0: control, no flooding; W1: 2.0-3.0 cm flooding; W2: 3.0-4.0 cm flooding; CK: control, no ultrasonic treatment; S1: ultrasonic waves in the dry state; S2: ultrasonic waves in the wet state. 


\section{Ultrasonic treatment provoked regulation of seedling growth}

Leaves under waterlogging stress were smaller than those in W0, but ultrasonic treatments notably improved total leaf area in both cultivars (Figure 2). The total leaf area of W0S1 and W0S2 improved by $29.09 \%$ and $37.41 \%$ in YJSM, respectively, and $14.59 \%$ and $18.58 \%$ in YXYZ, respectively. The improved situation showed that total leaf areas S1 and S2 improved under W1 and W2 in YJSM. Furthermore, when compared with W1CK, W1S1 was enhanced by $13.19 \%$ and W1S2 improved by $31.30 \%$ in YXYZ. The total leaf area of W2S1 increased by $13.48 \%$ compared with W2CK, but there was nonsignificant difference between W2S1 and W2CK.

The ultrasonic treatment of S1 markedly regulated seedling height under waterlogging stress in YJSM, but there was nonsignificant difference among ultrasonic treatments under waterlogging stress in YXYZ (P > 0.05) (Figure 3). Compared with W0CK, the height of W0S1 was $15.99 \%$ greater. The height in W1S1 and W1S2 significantly increased by $47.15 \%$ and $30.77 \%$, respectively, compared with W1CK. Meanwhile, W2S1 and W2S2 were higher than W2CK and reached a significant level in YJSM. Seedling height in S1 and S2 was higher than in CK under either W1 or W2; however, this did not significantly differ from the ultrasonic treatment under either W1 or W2 in YXYZ. For example, W2S1 was $13.31 \%$ higher than W2CK when W2S2 was $22.37 \%$ higher than W2CK.

Figure 2. Effect of ultrasonic and waterlogging treatments on seedling total leaf area.

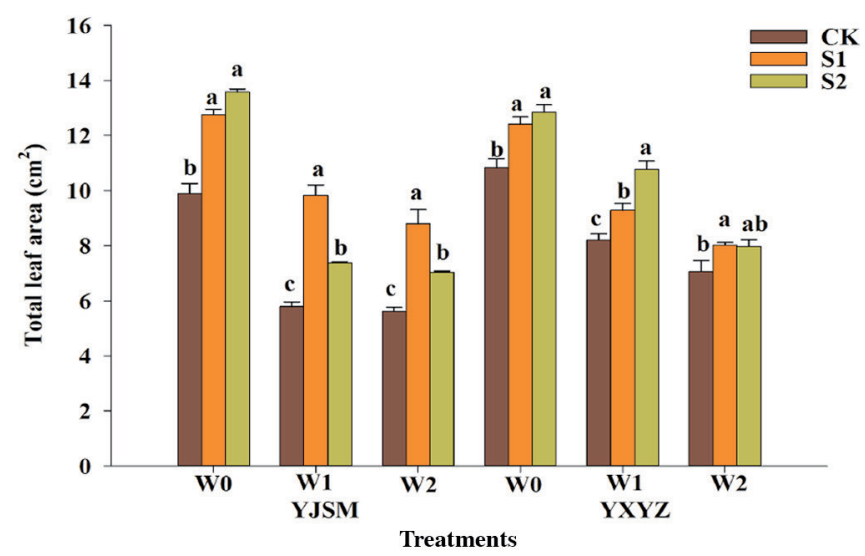

Different letters in a column indicate differences according to the LSD test $(\mathrm{P}<0.05)$.

YJSM: Yuejingsimiao; YXYZ: Yuxiangyouzhan; W0: control, no flooding; W1: 2.0-3.0 cm flooding; W2: 3.0-4.0 cm flooding; CK: control, no ultrasonic treatment; S1: ultrasonic waves in the dry state; S2: ultrasonic waves in the wet state.

Figure 3. Effect of ultrasonic and waterlogging treatments on seedling height.

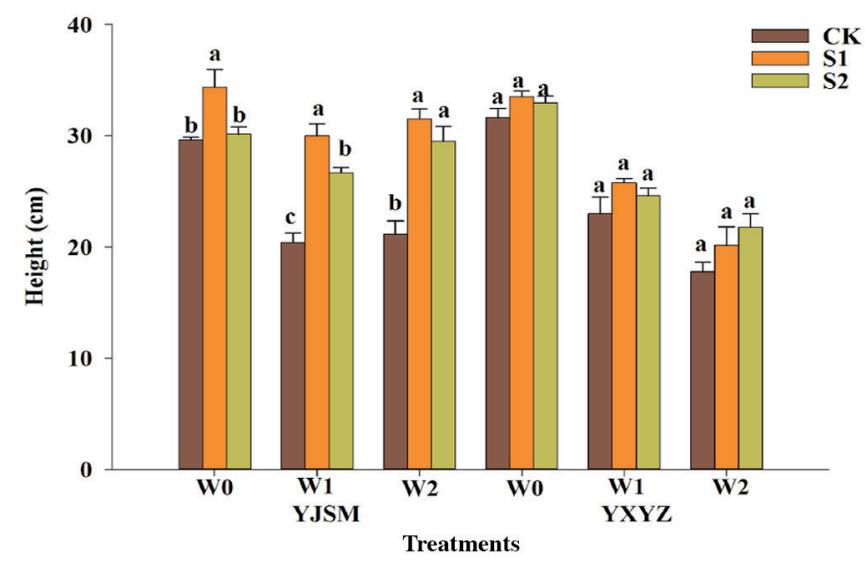

Different letters in a column indicate differences according to the LSD test $(\mathrm{P}<0.05)$.

YJSM: Yuejingsimiao; YXYZ: Yuxiangyouzhan; W0: control, no flooding; W1: 2.0-3.0 cm flooding; W2: 3.0-4.0 cm flooding; CK: control, no ultrasonic treatment; S1: ultrasonic waves in the dry state; S2: ultrasonic waves in the wet state. 
Ultrasonic treatments significantly improved seedling basal diameter under waterlogging stress for both cultivars, and seedlings under W1 and W2 had a lower basal diameter than W0 (Figure 4). For YJSM, the basal diameter in W0S2 increased by $25.12 \%$ compared with W0CK; however, W1S1 and W1S2 were $70.49 \%$ and $94.26 \%$ higher than W0CK, and a similar trend was observed in S1 and S2 under W2. For YXYZ, there was a $33.59 \%$ and $64.46 \%$ increase under WOS1 and W0S2, respectively, compared with W0CK. Otherwise, basal diameters were significantly wider in W2S1 and W2S2 than in W2CK.

\section{Ultrasonic treatment improved seedling weight}

Fresh and dry weight significantly increased with the ultrasonic treatments under waterlogging stress in both cultivars, and S1 under waterlogging stress had a greater ability to improve seedling weight (Table 1). For fresh weight, W0CK was $18.54 \%$ and $4.44 \%$ significantly lower than WOS1 and W0S2, respectively. In addition, values for W1S1 and W1S2 increased by $43.40 \%$ and $39.71 \%$, respectively, under low waterlogging stress. At the same time, W2CK had the lowest fresh weight and increased by $54.21 \%$ and $11.01 \%$ compared with W2S1 and W2S2, respectively. Similarly, dry weight under W0 and W2 waterlogging stress complied with fresh weight. The dry weight of W1S1 and W1S2 were 52.56\% and $36.89 \%$ higher, respectively, compared with W1CK.

For YXYZ, the dry weight of W0S2 improved by 54.78\%, while W1S1 (41.58\%) and W1S2 (122.19\%) were higher than W1CK. Fresh weight in the ultrasonic treatments under W0 and W1 performed similarly with dry weight, and W2S1 dry weight was $89.98 \%$ significantly higher than W2CK.

Table 1. Effects of ultrasonic and waterlogging treatments on seedling weight.

\begin{tabular}{|c|c|c|c|c|c|}
\hline \multirow{2}{*}{\multicolumn{2}{|c|}{ Treatments }} & \multicolumn{2}{|c|}{ YJSM } & \multicolumn{2}{|c|}{ YXYZ } \\
\hline & & \multirow{2}{*}{$\begin{array}{l}\text { Fresh weight } \\
0.55 \pm 0.02 \mathrm{~b}\end{array}$} & Dry weight & \multirow{2}{*}{$\begin{array}{l}\text { Fresh weight } \\
056+0.00 \mathrm{~b}\end{array}$} & $\begin{array}{l}\text { Dry weight } \\
\end{array}$ \\
\hline W0 & CK & & $0.083 \pm 0.001 b$ & & $0.057 \pm 0.003 b$ \\
\hline & $\mathrm{S} 1$ & $0.65 \pm 0.02 \mathrm{a}$ & $0.095 \pm 0.001 \mathrm{a}$ & $0.59 \pm 0.00 \mathrm{~b}$ & $0.057 \pm 0.001 b$ \\
\hline & $\mathrm{S} 2$ & $0.57 \pm 0.01 \mathrm{~b}$ & $0.081 \pm 0.001 b$ & $0.66 \pm 0.02 \mathrm{a}$ & $0.088 \pm 0.003 \mathrm{a}$ \\
\hline \multirow[t]{3}{*}{ W1 } & CK & $0.34 \pm 0.02 b$ & $0.054 \pm 0.002 \mathrm{c}$ & $0.25 \pm 0.01 \mathrm{c}$ & $0.026 \pm 0.002 \mathrm{c}$ \\
\hline & S1 & $0.48 \pm 0.01 \mathrm{a}$ & $0.083 \pm 0.001 \mathrm{a}$ & $0.38 \pm 0.01 b$ & $0.037 \pm 0.002 b$ \\
\hline & $\mathrm{S} 2$ & $0.47 \pm 0.01 \mathrm{a}$ & $0.074 \pm 0.001 b$ & $0.47 \pm 0.01 \mathrm{a}$ & $0.058 \pm 0.002 \mathrm{a}$ \\
\hline \multirow[t]{3}{*}{ W2 } & CK & $0.32 \pm 0.00 \mathrm{c}$ & $0.040 \pm 0.001 \mathrm{c}$ & $0.13 \pm 0.00 c$ & $0.019 \pm 0.002 b$ \\
\hline & $\mathrm{S} 1$ & $0.50 \pm 0.01 \mathrm{a}$ & $0.078 \pm 0.001 \mathrm{a}$ & $0.34 \pm 0.01 \mathrm{a}$ & $0.037 \pm 0.002 \mathrm{a}$ \\
\hline & $\mathrm{S} 2$ & $0.36 \pm 0.01 \mathrm{~b}$ & $0.065 \pm 0.001 b$ & $0.23 \pm 0.01 b$ & $0.023 \pm 0.001 b$ \\
\hline
\end{tabular}

Different letters in a column indicate significant differences according to the LSD test $(\mathrm{P}<0.05)$. YJSM: Yuejingsimiao; YXYZ: Yuxiangyouzhan; W0: control, no flooding; W1: 2.0-3.0 cm flooding; W2: 3.0-4.0 cm flooding; CK: control, no ultrasonic treatment; S1: ultrasonic waves in the dry state; S2: ultrasonic waves in the wet state.

Figure 4. Effect of ultrasonic and waterlogging treatments on seedling basal diameter.

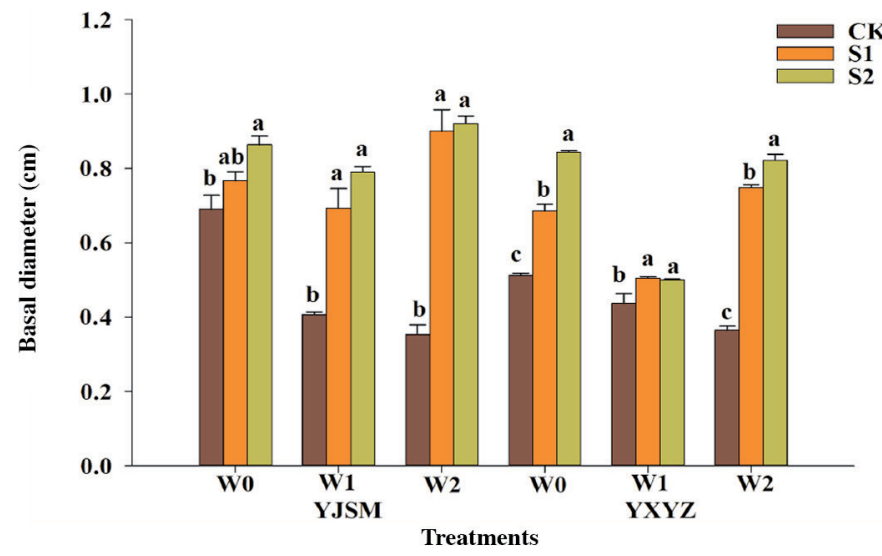

Different letters in a column indicate differences according to the LSD test $(\mathrm{P}<0.05)$.

YJSM: Yuejingsimiao; YXYZ: Yuxiangyouzhan; W0: control, no flooding; W1: 2.0-3.0 cm flooding; W2: 3.0-4.0 cm flooding; CK: control, no ultrasonic treatment; S1: ultrasonic waves in the dry state; S2: ultrasonic waves in the wet state. 


\section{Number of roots under ultrasonic treatment}

Ultrasonic treatments improved the number of both white roots and total roots per unit seedling in both cultivars (Table 2). The number of total roots under WOS1 and WOS2 increased $18.39 \%$ and 30.12\% in YJSM, respectively, while W0S2 was $17.65 \%$ higher than W0CK in YXYZ. Likewise, W1S1 significantly decreased by $9.81 \%$ compared with W1CK; however, W1S2 was $13.58 \%$ higher in YJSM. On the contrary, W1S1 improved by $21.40 \%$ compared with W1CK in YXYZ. The number of total roots for W2S1 was $20.57 \%$ and $31.65 \%$ higher, respectively, than for W2CK in both cultivars, but there was nonsignificant difference between W2S2 and W2CK in YXYZ. The number of white roots under W1S2 was 53.85\% higher than W1CK in YJSM and 37.41\% in YXYZ. Nonsignificant difference was observed between ultrasonic treatments under W2 waterlogging stress in YJSM; however, the number of white roots under W2S1 and W2S2 increased by $22.90 \%$ and $42.75 \%$, respectively, in YXYZ.

\section{Effect of the ultrasonic treatment on SPAD values}

The SPAD values for the ultrasonic treatments increased in both cultivars, and these reached significant differences under waterlogging stress for S1 and S2 in YJSM (Figure 5). The increasing trend was similar for YJSM; S1 and S2 were significantly higher than CK under waterlogging treatments. For example, the increase in W1S1 and W1S2 was significant with values of $8.40 \%$ and $8.53 \%$, respectively, compared with W1CK, while W2S1 and W2S2 increased $23.55 \%$ and $20.59 \%$, respectively, compared with W2CK. The SPAD value for YXYZ differed from ultrasonic treatments under waterlogging. The WOS1 treatment value was $8.26 \%$ compared with W0CK, while W2S1 and W2S2 improved by $6.95 \%$ and $9.40 \%$, respectively, compared with W2CK.

Table 2. Effects of ultrasonic and waterlogging treatments on the number of roots.

\begin{tabular}{rrrrrrr}
\hline & \multicolumn{2}{c}{ YJSM } & & \multicolumn{2}{c}{ YXYZ } \\
\cline { 3 - 4 } \cline { 6 - 7 } Treatments & White roots & Total roots & & White roots & \multicolumn{1}{c}{ Total roots } \\
\hline W0 & CK & $3.67 \pm 0.22 \mathrm{a}$ & $6.92 \pm 0.36 \mathrm{~b}$ & & $5.39 \pm 0.20 \mathrm{ab}$ & $9.92 \pm 0.22 \mathrm{~b}$ \\
& S1 & $3.64 \pm 0.07 \mathrm{a}$ & $8.19 \pm 0.30 \mathrm{a}$ & & $4.72 \pm 0.15 \mathrm{~b}$ & $10.17 \pm 0.36 \mathrm{~b}$ \\
& S2 & $2.89 \pm 0.18 \mathrm{~b}$ & $9.00 \pm 0.25 \mathrm{a}$ & & $6.00 \pm 0.29 \mathrm{a}$ & $11.67 \pm 0.22 \mathrm{a}$ \\
W1 & CK & $2.53 \pm 0.12 \mathrm{~b}$ & $7.36 \pm 0.07 \mathrm{~b}$ & & $3.86 \pm 0.27 \mathrm{~b}$ & $8.31 \pm 0.19 \mathrm{c}$ \\
& S1 & $3.00 \pm 0.14 \mathrm{~b}$ & $6.64 \pm 0.22 \mathrm{c}$ & & $5.72 \pm 0.15 \mathrm{a}$ & $10.08 \pm 0.21 \mathrm{a}$ \\
& S2 & $3.89 \pm 0.18 \mathrm{a}$ & $8.36 \pm 0.07 \mathrm{a}$ & & $5.31 \pm 0.19 \mathrm{a}$ & $9.06 \pm 0.24 \mathrm{~b}$ \\
W2 & CK & $2.50 \pm 0.14 \mathrm{a}$ & $5.25 \pm 0.14 \mathrm{~b}$ & & $3.64 \pm 0.22 \mathrm{c}$ & $6.92 \pm 0.46 \mathrm{~b}$ \\
& S1 & $2.44 \pm 0.15 \mathrm{a}$ & $6.33 \pm 0.22 \mathrm{a}$ & & $4.47 \pm 0.12 \mathrm{~b}$ & $9.11 \pm 0.18 \mathrm{a}$ \\
& S2 & $2.42 \pm 0.13 \mathrm{a}$ & $6.25 \pm 0.14 \mathrm{a}$ & & $5.19 \pm 0.10 \mathrm{a}$ & $7.53 \pm 0.24 \mathrm{~b}$ \\
\hline
\end{tabular}

Different letters in a column indicate differences according to the LSD test $(\mathrm{P}<0.05)$.

YJSM: Yuejingsimiao; YXYZ: Yuxiangyouzhan; W0: control, no flooding; W1: $2.0-3.0 \mathrm{~cm}$ flooding; W2: 3.0-4.0 cm flooding; CK: control, no ultrasonic treatment; S1: ultrasonic waves in the dry state; S2: ultrasonic waves in the wet state.

Figure 5. Effect of ultrasonic and waterlogging treatments on SPAD value.

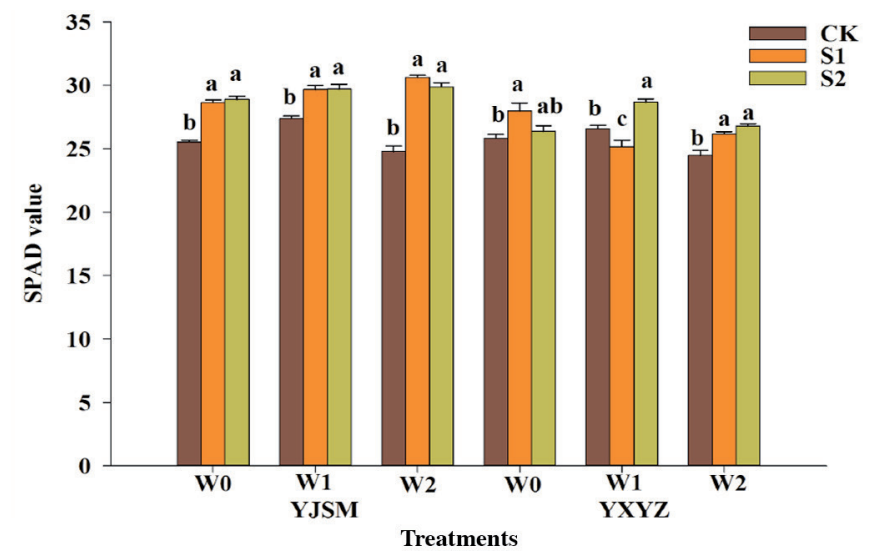

Different letters in a column indicate differences according to the LSD test $(\mathrm{P}<0.05)$.

YJSM: Yuejingsimiao; YXYZ: Yuxiangyouzhan; W0: control, no flooding; W1: 2.0-3.0 cm flooding; W2: 3.0-4.0 cm flooding; CK: control, no ultrasonic treatment; S1: ultrasonic waves in the dry state; S2: ultrasonic waves in the wet state. 


\section{Ultrasound induced antioxidant responses}

Significant increases in antioxidant enzyme activity were observed in ultrasonic treatments that were also affected by the degree of waterlogging stress (Table 3). The POD activity for W1S1 was the highest among W1CK, W1S1, and W1S2 in both cultivars, and there was a similar trend in YJSM under W2. The POD activity for WOS1 and WOS2 increased by $39.62 \%$ and $10.43 \%$, respectively, compared with W0CK: the W1S1 treatment was $22.17 \%$ higher than W1CK for YJSM. Meanwhile, when comparing with W1CK, the W1S1 treatment significantly increased POD activity by $21.32 \%$ compared with W2CK and W2S2 was $52.52 \%$ higher in YXYZ.

There was nonsignificant difference in YJSM for CAT activity among W0CK, WOS1, and WOS2. On the contrary, CAT activity for W1S1 and W1S2 was $11.74 \%$ and $7.87 \%$ higher, respectively, compared with W1CK, whereas W2S1 and W2S2 had values of $18.49 \%$ and $26.37 \%$, respectively, compared to W2CK. For YXYZ, S2 significantly differed from CK under W0 and was similarly coordinated with those under W2. Compared with W1CK, CAT activity was significantly enhanced for W1S1 and W1S2 by $15.02 \%$ and $43.29 \%$, respectively (Table 3).

\section{Ultrasonic treatment positively affected osmoregulation}

The MDA content also increased with the increasing degree of flooding. For the same flooding treatment, different ultrasonic treatments reduced MDA content and nonsignificant differences were observed between W0CK and W0S2 for both cultivars (Figure 6). The MDA content of W1S1 was 19.87\% lower than W1CK, and reductions of $25.83 \%$ and $20.92 \%$ occurred under W2S1 and W2S2, respectively, for YJSM compared with W2CK. The MDA content for W1S1 and W1S2 significantly decreased by $31.84 \%$ and $38.74 \%$, respectively; similarly, W2S1 and W2S2 were lower than W2CK by $23.85 \%$ and $32.19 \%$, respectively.

The ultrasonic treatments had a clear impact on the soluble protein content under waterlogging stress, especially for YXYZ (Figure 7). For YJSM, the soluble protein content of S2 significantly decreased by $2.34 \%$ and $3.39 \%$ under W0 and $\mathrm{W} 1$, respectively, compared with CK; however, W2S1 and W2S2 were higher than CK by $3.16 \%$ and $5.50 \%$, respectively. When comparing with W0CK for YXYZ, contents in W0S1 increased by $31.83 \%$, but there was nonsignificant difference between W0CK and W0S2. In addition, W1S1 was 37.10\% and W1S2 was 57.10\% higher than CK under W1. However, when compared with W2CK, W2S2 decreased by $8.84 \%$.

\section{Multivariate analysis}

The correlations between dry weight per seedling and other studied parameters are shown in Table 4 . There was a significant positive correlation between dry weight and seedling emergence rate, fresh weight, leaf area, and height in both cultivars. There was a significant positive correlation between dry weight and basal diameter in YJSM, and a significant negative correlation between dry weight and MDA content in YJSM. Likewise, seeding rate was significantly and positively correlated with dry weight for YXYZ. A significant positive correlation between dry weight and the number of total roots and white roots in YXYZ was found (Table 4).

Table 3. Effects of ultrasonic and waterlogging treatments on antioxidant enzyme activity.

\begin{tabular}{|c|c|c|c|c|c|}
\hline \multirow{2}{*}{\multicolumn{2}{|c|}{ Treatments }} & \multicolumn{2}{|c|}{ YJSM } & \multicolumn{2}{|c|}{ YXYZ } \\
\hline & & POD & CAT & POD & CAT \\
\hline \multirow{3}{*}{ W0 } & & $\mathrm{U} \mathrm{g}^{-1} \min ^{-1}$ & $\mathrm{U} \mathrm{mg}^{-1} \mathrm{~s}^{-1}$ & $\mathrm{U} \mathrm{g}^{-1} \min ^{-1}$ & $\mathrm{U} \mathrm{mg}^{-1} \mathrm{~s}^{-1}$ \\
\hline & CK & $165.83 \pm 7.20 \mathrm{c}$ & $307.28 \pm 7.97 \mathrm{a}$ & $128.97 \pm 4.58 b$ & $236.86 \pm 6.94 b$ \\
\hline & S1 & $231.54 \pm 1.46 \mathrm{a}$ & $314.24 \pm 6.51 \mathrm{a}$ & $156.82 \pm 5.68 \mathrm{a}$ & $248.78 \pm 7.54 b$ \\
\hline & $\mathrm{S} 2$ & $183.13 \pm 3.40 \mathrm{~b}$ & $308.07 \pm 4.16 \mathrm{a}$ & $162.43 \pm 3.64 a$ & $313.58 \pm 13.66 a$ \\
\hline \multirow[t]{2}{*}{ W1 } & $\mathrm{CK}$ & $183.52 \pm 6.24 b$ & $308.80 \pm 1.79 b$ & $170.70 \pm 5.41 b$ & $318.45 \pm 8.82 \mathrm{c}$ \\
\hline & $\mathrm{S} 1$ & $224.20 \pm 10.98 \mathrm{a}$ & $345.07 \pm 5.25 \mathrm{a}$ & $207.10 \pm 5.92 \mathrm{a}$ & $366.30 \pm 12.44 b$ \\
\hline \multirow{4}{*}{ W2 } & $\mathrm{S} 2$ & $193.68 \pm 2.74 b$ & $333.12 \pm 2.43 \mathrm{a}$ & $171.18 \pm 4.00 \mathrm{~b}$ & $456.31 \pm 14.43 a$ \\
\hline & CK & $180.29 \pm 5.32 b$ & $268.49 \pm 1.95 c$ & $134.75 \pm 4.93 b$ & $324.26 \pm 3.24 b$ \\
\hline & S1 & $218.70 \pm 8.99 a$ & $318.14 \pm 7.95 b$ & $152.07 \pm 5.84 b$ & $309.55 \pm 7.37 \mathrm{~b}$ \\
\hline & $\mathrm{S} 2$ & $193.62 \pm 3.58 b$ & $339.28 \pm 5.82 \mathrm{a}$ & $205.53 \pm 6.86 a$ & $381.26 \pm 8.28 \mathrm{a}$ \\
\hline
\end{tabular}

Different letters in a column indicate significant differences according to the LSD test $(\mathrm{P}<0.05)$. YJSM: Yuejingsimiao; YXYZ: Yuxiangyouzhan; POD: peroxidase; CAT: catalase; W0: control, no flooding; W1: 2.0-3.0 cm flooding; W2: 3.0-4.0 cm flooding; CK: control, no ultrasonic treatment; S1: ultrasonic waves in the dry state; S2: ultrasonic waves in the wet state. 
Figure 6. Effect of ultrasonic and waterlogging treatments on malondialdehyde (MDA) content.

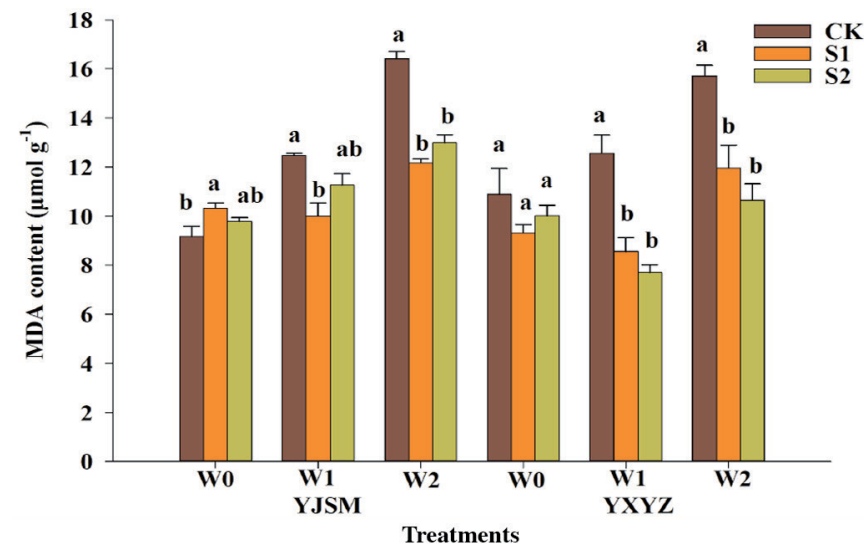

Different letters in a column indicate differences according to the LSD test $(\mathrm{P}<0.05)$.

YJSM: Yuejingsimiao; YXYZ: Yuxiangyouzhan; W0: control, no flooding; W1: 2.0-3.0 cm flooding; W2: 3.0-4.0 cm flooding; CK: control, no ultrasonic treatment; S1: ultrasonic waves in the dry state; S2: ultrasonic waves in the wet state.

Figure 7. Effect of ultrasonic and waterlogging treatments on soluble protein content.

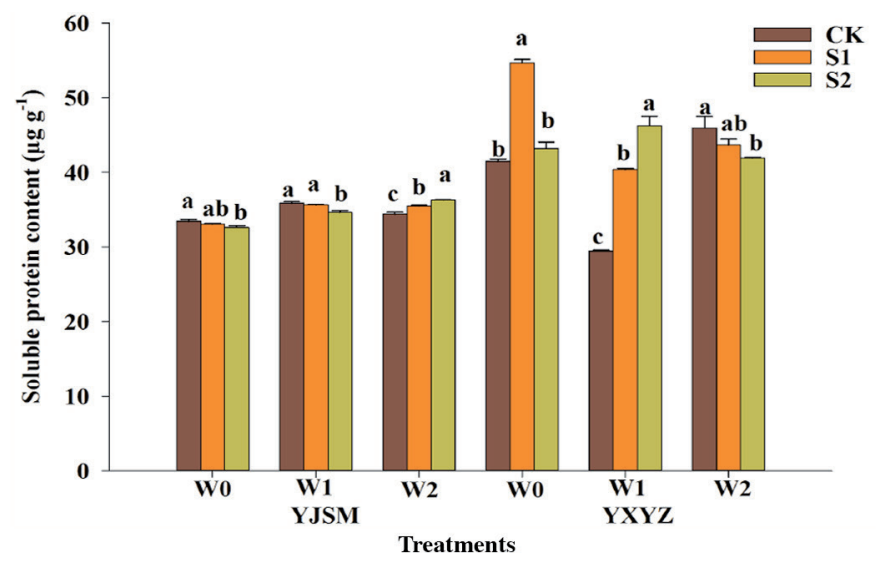

Different letters in a column indicate differences according to the LSD test $(\mathrm{P}<0.05)$.

YJSM: Yuejingsimiao; YXYZ: Yuxiangyouzhan; W0: control, no flooding; W1: 2.0-3.0 cm flooding; W2: 3.0-4.0 cm flooding; CK: control, no ultrasonic treatment; S1: ultrasonic waves in the dry state; S2: ultrasonic waves in the wet state.

Table 4. Correlations between dry weight per seedling and studied parameters.

\begin{tabular}{lcccc}
\hline Parameters & YJSM & P-Value & YXYZ & P-Value \\
\hline Fresh weight & 0.9228 & 0.0004 & 0.9463 & 0.0001 \\
Seedling emergence rate & 0.4930 & 0.0090 & 0.8811 & 0.0017 \\
Leaf area & 0.8392 & 0.0047 & 0.9355 & 0.0002 \\
SPAD value & 0.4911 & 0.1794 & 0.3986 & 0.2879 \\
Height & 0.9044 & 0.0008 & 0.8277 & 0.0059 \\
Basal diameter & 0.6952 & 0.0376 & 0.4179 & 0.2630 \\
Number of total roots & 0.6024 & 0.0860 & 0.6904 & 0.0395 \\
Number of white roots & 0.5894 & 0.0949 & 0.8853 & 0.0015 \\
POD & 0.5034 & 0.1671 & -0.2029 & 0.6007 \\
CAT & 0.5430 & 0.1309 & -0.1643 & 0.6727 \\
MDA & -0.8618 & 0.0000 & -0.5657 & 0.1124 \\
Soluble protein & -0.4303 & 0.2476 & 0.3335 & 0.3805 \\
\hline
\end{tabular}

YJSM: Yuejingsimiao; YXYZ: Yuxiangyouzhan; POD: peroxidase; CAT: catalase; MDA: malondialdehyde. 
In order to examine in depth the possible relationship between the studied parameters, a heatmap was created (Figure 8a). It was classified in two large groups, the MDA content and the other parameters; there was a significant negative correlation between these two groups. The heatmap also revealed correlations for dry weight, leaf area, soluble protein content, fresh weight, height, number of total (white) roots, and seedling emergence rate (Figure 8a). Furthermore, compounds are correlated with dry weight per seedling in Figure 8b. Fresh weight, height, SPAD values, basal diameter, and leaf area were the top five parameters that were strongly correlated with dry weight (Figure 8b).

\section{DISCUSSION}

In vivo and in vitro gas exchange $\left(\mathrm{O}_{2}\right.$ and $\left.\mathrm{CO}_{2}\right)$ between plants and the underground environment could be obstructed by excess water under waterlogging conditions; the physiological mechanism was basically affected, resulting in growth disorders (Ashraf et al., 2017). Obstacles such as limited oxygen, severely restricted aerobic respiration, and loss of ATP for growth caused apoptosis and necrosis, leading to plant death. Membrane lipids were aggravated by the production and accumulation of $\mathrm{H}_{2} \mathrm{O}_{2}$, one of the oxygen free radicals that influences the regulation of plant growth (Chen et al., 2013). The impact of cavitation, thermal effect, and mechanical mass transfer are acceptable factors of ultrasound to stimulate crops even though the botanical mechanisms are intricate (Xie et al., 2009).

Ultrasound at lower doses over an average time could stimulate cell division and seedlings would still have a good quality. Its effectiveness depends on frequency and intensity (Machikowa et al., 2013). The S2 treatment enhanced the seedling emergence rate, leaf area, and basal diameter, thus improving seedling quality (Figures 1,2, and 4). One possible explanation for this result is that ultrasonic waves going through the seeds modify the permeability of the cell wall and membrane by acoustic cavitation; this increases seed porosity beneficial for seed absorption and consumption of water and oxygen (Sirsi and Borden, 2012). Therefore, sufficient oxygen can be used by cell proliferation, thereby stimulating seedling growth.

Figure 8. Correlations of the studied parameters. Correlation heatmap (a) and compounds correlated with dry weight (b).
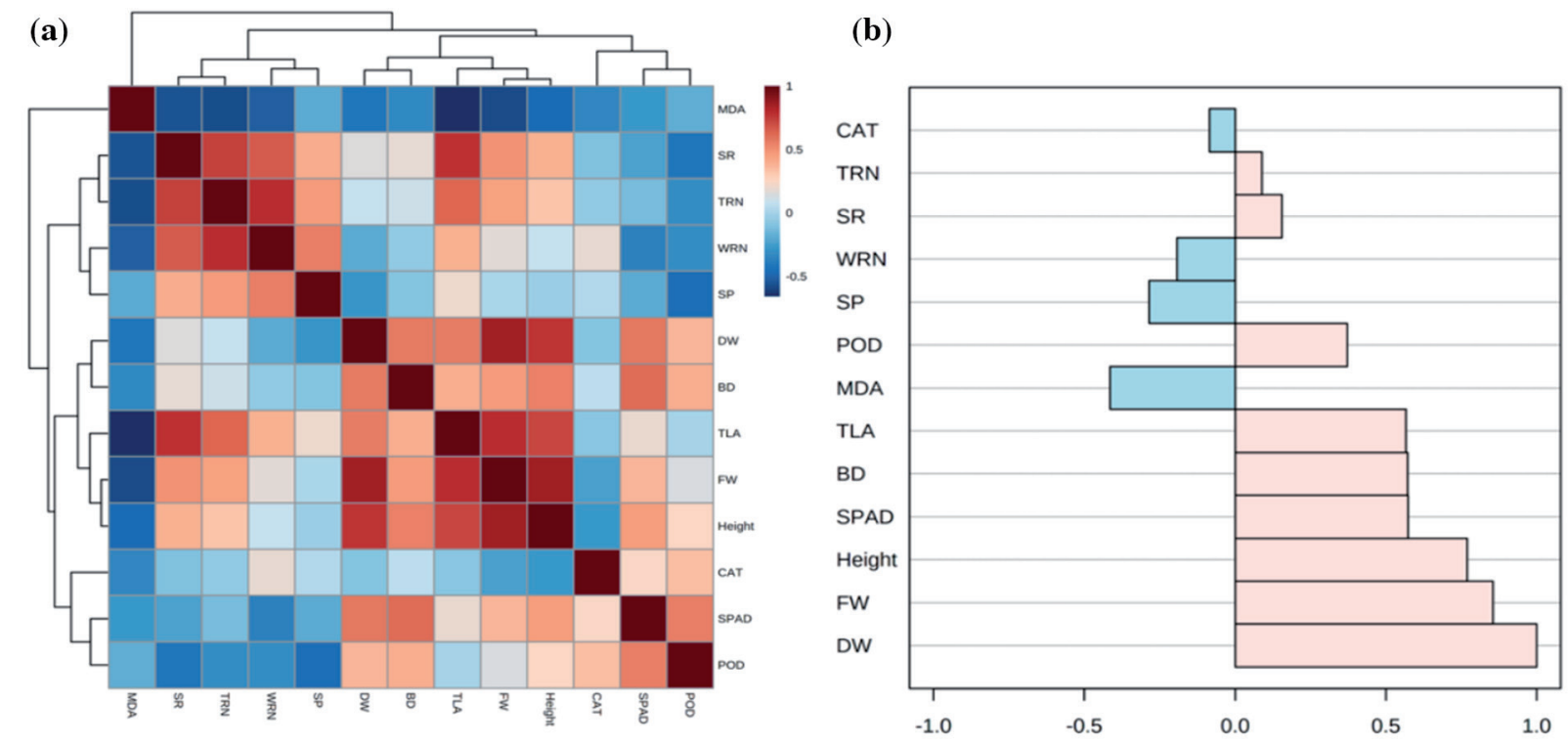

CAT: Catalase activity; TRN: number of total roots; SR: seedling emergence rate; WRN: number of white roots; SP: soluble protein content; POD: peroxidase; MDA: malondialdehyde content; TLA: total leaf area; BD: basal diameter; FW: fresh weight per seedling; DW: dry weight per seedling. 
When rice is subjected to biotic or abiotic stress, protective defenses are activated, including POD, CAT, and osmotic regulation in plants. The ROS has a dual effect on plants, whose excessive accumulation induces oxidative damage that leads to cell death as a side effect (Wang et al., 2015; Yan et al., 2017). In our study, we found that ultrasonic treatments increased POD and CAT activities under waterlogging stress, which provide an effective protection against the ROS (Table 3). The MDA, as a decomposition product of lipid peroxidation, disintegrates the cell membrane, damages functional molecules, and interferes with the metabolism. Studies have reported that MDA increased under atmospheric pressure, was reduced and resulted in a higher MDA content for W0 than for W1 or W2 (Figure 6) (Xiong et al., 2013).

The rational use of ultrasound contributes to extracting phenolic compounds, resulting in a strengthened antioxidant defense system to prevent $\mathrm{H}_{2} \mathrm{O}_{2}$-induced cell death (Yingngam et al., 2014). Seedling height, weight, and number of roots increased for S1 and S2 compared with the control (Figure 3, Tables 1 and 2), which was consistent with a previous study (Wang et al., 2012). It was reported that ultrasound helped to eliminate free radicals by increasing POD activity and soluble protein content, decreasing MDA content and electrolyte leakage, and eventually enhance seed vigor and plant quality (Chen et al., 2013). Ultrasonic waves contribute to plant growth, and this can change the physiological and biochemical aspects of seeds; they can activate antioxidant enzymes to resist various stresses (Teixeira Da Silva and Dobránszki, 2014). Furthermore, the ultrasonic treatment of seeds improves crop quality; it can be an essential practice to reduce the residues from chemical pesticides and fertilizers which has decreased rice yield in recent years (Chen et al., 2016).

The present study showed that the ultrasonic treatment strengthened antioxidant activities and seedlings, resulting in strong resistance against waterlogging stress, which was associated with findings by Kong et al. (2017). There is high-power ultrasonic wave energy in plant organogenesis (Teixeira Da Silva and Dobránszki, 2014). Seedlings in the ultrasonic treatment had better seedling emergence rate, height, weight, and POD activity (Tables 1 and 3, Figures 1 and 3). This could be due to the fact that, when treated by ultrasound at the appropriate frequency, the energy of the waves could be absorbed by the seeds. This could stimulate the cells to raise their enzymatic activities and change their molecular structure to soften the seed coat to efficiently absorb nutrients. The ultrasound treatment of seeds usually enhances the seedling emergence rate, promotes seedling growth, and can result in better rice quality and yield.

\section{CONCLUSIONS}

The ultrasonic treatment of wet seeds significantly increased the seedling emergence rate under waterlogging stress. There were also significant effects on seedling quality and peroxidase and catalase activities. Improvement in the protective enzyme activity might decrease the damage caused under waterlogging conditions.

\section{ACKNOWLEDGEMENTS}

Funding was provided by the National Natural Science Foundation of China (31271646). The World Bank Loan Agricultural Pollution Control Project in Guangdong (0724-1510A08N3684) and the Technology System of Modern Agricultural Industry in Guangdong (2016LM1098) are also acknowledged for their contribution.

\section{REFERENCES}

Ashraf, U., Kanu, A.S., Deng, Q., Mo,Z., Pan, S., Tian, H., et al. 2017. Lead (Pb) toxicity; physio-biochemical mechanisms, grain yield, quality, and $\mathrm{Pb}$ distribution proportions in scented rice. Frontiers in Plant Science 8:259. doi:10.3389/fpls.2017.00259.

Chen, Y., Liu, Q., Yue, X., Meng, Z., and Liang, J. 2013. Ultrasonic vibration seeds showed improved resistance to cadmium and lead in wheat seedling. Environmental Science and Pollution Research 20:4807-4816. doi:10.1007/s11356-012-1411-1.

Chen, S., Xu, C., Yan, J., Zhang, X., Zhang, X., and Wang, D. 2016. The influence of the type of crop residue on soil organic carbon fractions: An 11-year field study of rice-based cropping systems in southeast China. Agriculture Ecosystem \& Environment 223:261-269. doi:10.1016/j.agee.2016.03.009.

Du, B., Luo, H., He, L., Zhang, L., Liu, Y., Mo, Z., et al. 2019. Rice seed priming with sodium selenate: Effects on germination, seedling growth, and biochemical attributes. Scientific Reports 9:4311. doi:10.1038/s41598-019-40849-3.

Fernández, R., Baschwitz, A., Reis, R., Mourato, M., Martins, L., and González, A. 2013. Growth and physiological responses to cadmium stress of two populations of Dittrichia viscosa (L.) Greuter. Journal of Hazardous Materials 244-245:555-562. doi:10.1016/j.jhazmat.2012.10.044. 
Ghobadi, M.E., Ghobadi, M., and Zebarjadi, A. 2017. Effect of waterlogging at different growth stages on some morphological traits of wheat varieties. International Journal of Biometeorology 61:635-645. doi:10.1007/s00484-016-1240-x.

Goussous, S.J., Samarah, N.H., Alqudah, A.M., and Othman, M.O. 2010. Enhancing seed germination of four crop species using an ultrasonic technique. Experimental Agriculture 46:231. doi:10.1017/S0014479709991062.

Groot, S.P.C., de Groot, L., Kodde, J., and van Treuren, R. 2015. Prolonging the longevity of ex situ conserved seeds by storage under anoxia. Plant Genetic Resources: Characterization and Utilization 13(1):18-26. doi:10.1017/S1479262114000586.

Groot, S.P.C., Surki, A.A., de Vos, R.C.H., and Kodde, J. 2012. Seed storage at elevated partial pressure of oxygen, a fast method for analysing seed ageing under dry conditions. Annals of Botany 110(6):1149-1159. doi:10.1093/aob/mcs198.

Kong, L., Ashraf, U., Cheng, S., Rao, G., Mo, Z., Tian, H., et al. 2017. Short-term water management at early filling stage improves early-season rice performance under high temperature stress in South China. European Journal of Agronomy 90:117-126. doi:10.1016/j.eja.2017.07.006.

Leon, A.D., Perera, R., Nittayacharn, P., Cooley, M., and Exner, A.A. 2018. Ultrasound contrast agents and delivery systems in cancer detection and therapy. Advances in Cancer Research 139:57-84. doi:10.1016/bs.acr.2018.04.002.

Luo, H.W., Du, B., Zheng, A.X., Lai, R.F., You, Z.S., Wang, M., et al. 2018. Flooding treatment restrains volunteer rice germination and seedling growth. Applied Ecology and Environmental Research 16:72317242. doi:http://dx.doi.org/10.5666/aeer/1605_72317242.

Machikowa, T., Kulratanarak, T., and Wonprasaid, S. 2013. Effects of ultrasonic treatment on germination of synthetic sunflower seeds. International Journal of Agricultural and Biological Engineering 7(1):1-3.

Miano,A.C., Forti, V.A.,Abud,H.F., Gomes-Junior,F.G., Cicero, S.M., and Augusto, P.E.D. 2015.Effect of ultrasound technology on barley seed germination and vigour. Seed Science and Technology 43:297-302. doi:10.15258/sst.2015.43.2.10.

Nishiuchi, S., Yamauchi, T., Takahashi, H., Kotula, L., and Nakazono, M. 2012. Mechanisms for coping with submergence and waterlogging in rice. Rice 5:2. doi:10.1186/1939-8433-5-2.

Rao, G., Ashraf, U., Huang, S., Cheng, S., Abrar, M., Mo, Z., et al. 2018. Ultrasonic seed treatment improved physiological and yield traits of rice under lead toxicity. Environmental Science and Pollution Research 25:3363733644. doi:10.1007/s11356-018-3303-5.

Rissel, D., Losch, J., and Peiter, E. 2014. The nuclear protein Poly (ADP-ribose) polymerase 3 (AtPARP3) is required for seed storability in Arabidopsis thaliana. Plant Biology 16:1058-1064. doi:10.1111/plb.12167.

Sairam, R.K., Dharmar, K., Chinnusamy, V., Lekshmy, S., Joshi, R., and Bhattacharya, P. 2011. NADPH oxidase as the source of ROS produced under waterlogging in roots of mung bean. Biologia Plantarum 55:741-746. doi:10.1007/s10535-011-0179-3.

Sirsi, S.R., and Borden, M.A. 2012. Advances in ultrasound mediated gene therapy using microbubble contrast agents. Theranostics 2:1208-1222. doi:10.7150/thno.4306.

Srivastava, N., Gonugunta, V.K., Puli, M.R., and Raghavendra, A.S. 2009. Nitric oxide production occurs downstream of reactive oxygen species in guard cells during stomatal closure induced by chitosan in abaxial epidermis of Pisum sativum. Planta 229:757-765. doi:10.1007/s00425-008-0855-5.

Teixeira Da Silva, J.A., and Dobránszki, J. 2014. Sonication and ultrasound: impact on plant growth and development. Plant Cell, Tissue and Organ Culture 117:131-143. doi:10.1007/s11240-014-0429-0.

Wang, Q., Chen, G., Yersaiyiti, H., Liu, Y., Cui, J., Wu, C., et al. 2012. Modeling analysis on germination and seedling growth using ultrasound seed pretreatment in switchgrass. PLOS ONE 7:10. doi:10.1371/journal.pone.0047204.

Wang, Y., Li, Y., Xue, H., Pritchard, H.W., and Wang, X. 2015. Reactive oxygen species-provoked mitochondria-dependent cell death during ageing of elm (Ulmus pumila L.) seeds. Plant Journal 81:438-452. doi:10.1111/tpj.12737.

Wojtyla, A., Lechowska, K., Kubala, S., and Garnczarska, M. 2016. Different modes of hydrogen peroxide action during seed germination. Frontiers in Plant Science 7:66. doi:10.3389/fpls.2016.00066.

Xie, B., Liu, H., and Yan, Y. 2009. Improvement of the activity of anaerobic sludge by low-intensity ultrasound. Journal of Environmental Management 90:260-264. 10.1016/j.jenvman.2007.09.004.

Xiong, J., Hu, S., Li, J., He, S., and Feng, L. 2013. Influence of strong electric field on MDA and SOD of rice under atmosphere pressure. Journal of Physics Conference 418:012141. doi:10.1088/1742-6596/418/1/012141.

Yan, J., Tong, T., Li, X., Chen, Q., Dai, M., Niu, F., et al. 2017. A novel NAC-type transcription factor, NAC87, from oilseed rape modulates reactive oxygen species accumulation and cell death. Plant \& Cell Physiology 59(2):290-303. doi:10.1093/pcp/pcx184.

Yingngam, B., Monschein, M., and Brantner, A. 2014. Ultrasound-assisted extraction of phenolic compounds from Cratoxylum formosum ssp. formosum leaves using central composite design and evaluation of its protective ability against $\mathrm{H}_{2} \mathrm{O}_{2}$-induced cell death. Asian Pacific Journal of Tropical Medicine 71:S497-S505. doi:10.1016/S1995-7645(14)60281-9. 